\title{
Endometrial Tumor Classification by Histomorphology and Biomarkers in the Nurses' Health Study
}

\author{
Jaclyn C. Watkins $\mathbb{D}^{1}{ }^{1}$ Michael J. Downing, ${ }^{1}$ Marta Crous-Bou, ${ }^{2,3}$ Evan L. Busch, ${ }^{2,4}$ \\ Maxine M. Chen, ${ }^{2,4,5}$ Immaculata De Vivo, ${ }^{2,4,5}$ and George L. Mutter $\mathbb{D}^{1,5}$ \\ ${ }^{1}$ Department of Pathology, Brigham and Women's Hospital, 75 Francis Street, Boston, MA, USA 02115 \\ ${ }^{2}$ Department of Epidemiology, Harvard T.H. Chan School of Public Health, 677 Huntington Ave., Boston, MA, USA 02115 \\ ${ }^{3}$ Unit of Nutrition and Cancer, Cancer Epidemiology Research Program, Catalan Institute of Oncology (ICO)-Bellvitge Biomedical \\ Research Institute (IDIBELL), L'Hospitalet de Llobregat, Barcelona 08908, Spain \\ ${ }^{4}$ Channing Division of Network Medicine, Brigham and Women's Hospital, 181 Longwood Avenue, Boston, MA, USA 02115 \\ ${ }^{5}$ Harvard Medical School, 25 Shattuck St., Boston, MA, USA 02115
}

Correspondence should be addressed to George L. Mutter; gmutter@partners.org

Received 14 September 2020; Revised 4 February 2021; Accepted 28 February 2021; Published 12 March 2021

Academic Editor: Samuel Antwi

Copyright (c) 2021 Jaclyn C. Watkins et al. This is an open access article distributed under the Creative Commons Attribution License, which permits unrestricted use, distribution, and reproduction in any medium, provided the original work is properly cited.

\begin{abstract}
Objective. Endometrial cancers have historically been classified by histomorphologic appearance, which is subject to interobserver disagreement. As molecular and biomarker testing has become increasingly available, the prognostic significance and accuracy of histomorphologic diagnoses have been questioned. To address these issues for a large, prospective cohort study, we provide the results of a centralized pathology review and biomarker analysis of all incidental endometrial carcinomas occurring between 1976 and 2012 in the Nurses' Health Study. Methods. Routine histology of all $(n=360)$ cases was reviewed for histomorphologic diagnosis. Cases were subsequently planted in a tissue microarray to explore expression of a variety of biomarkers (e.g., ER, PR, p53, PTEN, PAX2, AMACR, HNF1 $\beta$, Napsin A, p16, PAX8, and GATA3). Results. Histologic subtypes included endometrioid (87.2\%), serous $(5.6 \%)$, carcinosarcoma $(3.9 \%)$, clear cell $(1.7 \%)$, and mixed type $(1.7 \%)$. Biomarker results within histologic subtypes were consistent with existing literature: abnormal p53 was frequent in serous cases (74\%), and HNF1 $\beta$ (67\%), Napsin A (67\%), and AMACR (83\%) expression was frequent in clear cell carcinomas. Our dataset also allowed for examination of biomarker expression across non-preselected histologies. The results demonstrated that (1) HNF1 $\beta$ was not specific for clear cell carcinoma, (2) TP53 mutations occurred across many histologies, and (3) GATA3 was expressed across multiple histotypes, with $75 \%$ of positive cases demonstrating high-grade features. Conclusions. Our findings establish the subtypes of endometrial cancer occurring in the Nurses' Health Study, corroborate the sensitivity of certain well-established biomarkers, and call into question previously identified associations between certain biomarkers (e.g., HNF1B) and particular histotypes.
\end{abstract}

\section{Introduction}

Endometrial cancers have historically been subclassified by their histomorphologic appearance alone $[1,2]$. However, histomorphologic classification suffers from interobserver agreement issues, particularly in the case of high-grade endometrial carcinomas (e.g., FIGO grade 3 endometrioid, clear cell carcinoma, serous carcinoma, and carcinosarcoma) [3-7]. Further, in the molecular era, morphologic subtyping is considered insufficient for determining prognosis. Most famously, The Cancer Genome Atlas molecularly classified endometrial tumors into four categories which were prognostically significant irrespective of histomorphology [8].

Despite the inherent limitations in morphologic analysis of tumors, histomorphologic diagnosis largely determines inclusion in cancer registries as well as in clinical and epidemiologic research [9]. These diagnoses may suffer from changes in diagnostic categories over time, an absence of centralized pathology review, and, importantly, the absence of corroborative biomarker (immunohistochemical) data. 
The application of biomarkers (i.e., immunohistochemical staining) assists pathologists in rendering more accurate diagnoses. A recent study found that up to $40 \%$ of cases initially diagnosed as high-grade endometrial endometrioid adenocarcinomas may be reclassified as uterine serous carcinomas based on a panel of four immunostains (p53, p16, estrogen receptor (ER), and mammaglobin) [10]. Immunostains in routine practice, however, are often not pursued due to a pathologist's degree of morphologic certainty or the possibility of ambiguous results.

Further, biomarkers have their own limitations. Biomarker sensitivity and specificity have largely been determined by research conducted on highly selected cohorts of tumors, typically including only those with unambiguous morphologies. Thus, the sensitivity and specificity of markers tends to shift once the markers are applied in the clinical setting and to cases with less prototypical morphologies. For example, hepatocyte nuclear factor-1beta (HNF1 $\beta)$ expression was initially thought to have a specificity of 93 to $100 \%$ for clear cell carcinoma [11-14]; however, overtime, the specificity has been questioned as expression has been documented in a subset of serous and endometrioid carcinomas [15-17].

In this study, we attempt to address two of the aforementioned issues. First, we perform a centralized review of the incidental endometrial cancer cases arising in the Nurses' Health Study, a large, prospective cohort study with longterm follow-up. Secondly, we attempt to systematically apply a set of biomarkers to incidental endometrial cancer cases, thus exploring expression across a spectrum of subtypes. We explore biomarkers that are currently well-established in their interpretation (e.g., p53) as well as more novel biomarkers (e.g., GATA3). Further, we explore the limitations and benefits of applying biomarkers retrospectively in the case of a large epidemiologic study, especially the feasibility of interpretation in the setting of tissue microarrays.

\section{Materials and Methods}

This study was approved by the Human Studies Review Board at Brigham and Women's Hospital. The protocol for this study was approved by the Human Research Committees at Brigham and Women's Hospital, Boston, MA, USA.

2.1. Case Selection. Using data from the Nurses' Health Study $[18,19]$, a prospective cohort of 121,700 female nurses initiated in 1976, all incidental endometrial cancers diagnosed between 1976 and 2012 were identified.

When available, paraffin-embedded tissue and hematoxylin and eosin stained slides from the patient's endometrial biopsy or hysterectomy were obtained. Only cases with paraffin blocks for the creation of tissue microarrays were included. Full hematoxylin and eosin sections of the original clinical diagnostic blocks underwent central pathology review by a pathologist (GM), and a histologic diagnosis was rendered for each case using current diagnostic criteria [1]. Additional information regarding case ascertainment and tissue microarray construction has been published elsewhere [20].

For each specimen, a representative paraffin-embedded tissue block was chosen, and three $0.6 \mathrm{~mm}$ cores were planted in a tissue microarray. Serial sections of each microarray were used for marker studies as below.

2.2. Immunohistochemical Studies. For each marker studied, four-micron thick sections of each tissue microarray were stained in the following sequence: hematoxylin and eosin, marker replicate 1, pankeratin AE1/AE3 (Dako Cat\#M3515 at 1:200 dilution), and marker replicate 2 . The following immunostains were performed using the Leica Bond III staining platform using the following primary antibodies: estrogen receptor (ER, murine monoclonal ERID5 (Dako) at 1:300 dilution), progesterone receptor (PR, murine monoclonal PgR636 (Dako) at $1: 150$ dilution), p53 (murine monoclonal PAb 1801 (Leica Biosystems) at 1:300 dilution), AMACR (rabbit monoclonal Z2001 (Zeta Corp) at 1:50), ARID1A (rabbit polyclonal HPA005456 (Sigma) at 1:200 dilution), GATA3 (murine monoclonal L50-823 (Biocare) at $1: 100$ dilution), HNF1 $\beta$ (rabbit polyclonal HPA002083 (Sigma) at 1:500 dilution), Napsin A (murine monoclonal NCL-L-Napsin A (Leica) at 1:400 dilution), p16 (murine monoclonal E6H4 (Ventana) at 1:300 dilution), PAX2 (polyclonal rabbit Z-RX2 (InVitrogen) at 1:300 dilution), PAX8 (rabbit polyclonal 10336-1-AP (Proteintech) at 1:600 dilution), and PTEN (murine monoclonal 6h2.1 (Millipore) at $1: 100)$.

2.3. Marker Interpretation. All stained slides were digitally captured at $40 \mathrm{x}$ magnification by a Hamamatsu nanozoomer whole slide digital scanner. For each marker, hematoxylin and eosin and keratin-stained images of matched tissue microarray sections were used to confirm the presence of tumor tissue and to discriminate tumor cells from background as needed. Duplicate stains for each marker were independently scored for marker specific signal within tumor cells (JW and GM) (see Table 1 for scoring methodology). Discordant replicates were resolved by rereview. Markers scored on continuous scales were averaged across the duplicate runs for final data analysis. Technical failures due to tissue dropout, high background, or low signal were excluded as noninformative on a marker-by-marker basis.

Due to the limited tumor represented on the tissue microarrays, p53 was only interpreted as abnormal when staining was diffuse and strong; a "null" phenotype was not reported due to the possibility of failed staining or regional variability in tumor expression and thus the possibility of false positives [21].

2.4. Case Inclusion and Exclusion Criteria. Some patients had separate endometrial biopsies and hysterectomy specimens containing tumor. After staining, only unique specimens (1 per patient) were included in the final analysis, with preference for hysterectomy specimens over biopsies. Nonepithelial tumors were excluded. Carcinosarcoma and mixed carcinoma cases were included.

2.5. Covariates. Age at diagnosis (continuous) and body mass index at diagnosis [22] (continuous, $\mathrm{kg} / \mathrm{m}^{2}$ ) were assessed from the last biennial Nurses' Health Study questionnaire prior to cancer diagnosis. 
TABLE 1: Biomarker scoring and rationale for inclusion.

\begin{tabular}{|c|c|c|}
\hline Marker & Rationale & \#scoring \\
\hline AMACAR & Candidate clear cell type marker & $0=\mathrm{WT}$ (neg), $1=$ pos, $3=\mathrm{NI}$ \\
\hline ARID1A & Candidate clear cell type marker & $0=\mathrm{WT}($ pos $), 1=$ lost $($ neg $), 3=\mathrm{NI}$ \\
\hline Hnflbeta & Candidate clear cell type marker & $0=\mathrm{WT}($ neg), $1=$ pos, $3=\mathrm{NI}$ \\
\hline Napsin A & Candidate clear cell type marker & $0=\mathrm{WT}($ neg), $1=$ pos, $3=\mathrm{NI}$ \\
\hline p16 & Candidate serous type marker & $0=\mathrm{WT}$ (neg or patchy), $1=$ diffusely pos, $3=\mathrm{NI}$ \\
\hline ER & Hormone responsiveness & $\begin{array}{l}\text { Percentage positive nuclear staining: } 0=\text { neg }(<10 \% \text { staining }) \\
\qquad 1=\operatorname{pos}(\geq 10 \% \text { staining); also }\end{array}$ \\
\hline PR & Hormone responsiveness & $\begin{array}{l}\text { Percentage positive nuclear staining: } 0=\text { neg }(<10 \% \text { staining }) \text {, } \\
\qquad 1=\text { pos }(\geq 10 \% \text { staining })\end{array}$ \\
\hline PAX8 & Müllerian (paramesonephric) lineage marker & $0=$ neg, $1=$ pos, $3=\mathrm{NI}$ \\
\hline PAX2 & Primary inactivation mechanism & $0=\mathrm{WT}($ pos), $1=$ lost $($ neg), $3=\mathrm{NI}$ \\
\hline p53 & Primary mutation mechanism & $0=\mathrm{WT}, 1=$ diffuse, strong (mutated) expression $3=\mathrm{NI}$ \\
\hline PTEN & Primary mutation mechanism & $0=\mathrm{WT}($ pos), $1=$ lost $($ neg), $3=\mathrm{NI}$ \\
\hline GATA3 & Wolffian (mesonephric) lineage marker & $0=\mathrm{WT}($ neg), $1=$ pos, $3=\mathrm{NI}$ \\
\hline
\end{tabular}

${ }^{\#} \mathrm{WT}$ = wild type; $\mathrm{NI}$ = not informative; pos = positive; neg = negative.

2.6. Statistical Analyses. Statistical analysis was performed in SYSTAT (v13.1, Systat Software, Inc., San Jose, CA). Oneway ANOVA was used to independently compare age and body mass index with the diagnostic tumor subtypes. $t$-tests, with separate variances, were employed to compare biomarker expression across age and body mass index. Results with a $p$ value $<0.05$ were considered statistically significant. Hierarchical clustering of bimodally scored (normal vs. abnormal) marker results was performed using Ward's linkages and Jaccard similarity coefficient distance metric. Bimodal scoring of the continuous percentages of estrogen and progesterone receptor was done across a 10\% threshold.

\section{Results}

Pathology materials were received for 472 patients with reported endometrial cancer. After application of the inclusion criteria, 360 patients had adequate tissue for inclusion in the tissue microarray (343 hysterectomy specimens and 17 biopsy/curettages).

3.1. Histomorphologic Review. Histomorphologic diagnoses were as follows: 314 endometrioid (87.2\%), 20 serous (5.6\%), 14 carcinosarcoma (3.9\%), 6 clear cell (1.7\%), and 6 mixed endometrioid plus nonendometrioid type (1.7\%). Within the endometrioid group, 244 were grade 1, 43 were grade 2 , and 27 were grade 3 .

3.2. Biomarker Results within Histomorphologic Diagnoses (Table 2). Clear cell carcinomas frequently expressed AMACR, HNF1B, and Napsin A (83.3\%, 66.7\%, and 66.7\%, respectively). Serous carcinomas commonly displayed diffuse, strong p53 staining (mutant phenotype; 73.7\%) and p16 diffuse positivity (70\%). Endometrioid tumors demonstrated high rates of ER (92.7\%) and PR (85.1\%) expression, with the highest ER and PR expression noted in FIGO grade 1 endometrioid tumors.
3.3. Biomarker Expression across Histomorphologic Diagnoses. GATA3 was expressed across histotypes (16 cases total; 10 endometrioid (62.5\%), 3 serous (18.8\%), 2 carcinosarcoma $(12.5 \%)$, and 1 clear cell $(6.25 \%))$. GATA3 expression correlated positively with expression of $\operatorname{HNF} 1 \beta(p=0.009)$ and p16 $(p=0.017)$ and absence of staining for PAX2 $(p=0.002)$, ER $(p<0.001)$, and PR $(p<0.001)$. Rereview of the GATA3 positive tumors revealed an absence of morphologic findings consistent with mesonephric carcinomas. Further, 12 of the 16 cases showed high-grade morphology with ambiguous cytology (Figure 1). GATA3 and PAX8, respectively, markers of mesonephric and Müllerian origin, were not always mutually exclusive. $79.6 \%$ of cases (262/329) were positive for only one of the two markers, $15.8 \%$ (52/329) were negative for both, and $4.6 \%$ of cases (15/329) were positive for both.

Diffuse, strong p53 expression (mutant phenotype) was seen across histotypes. Of p53 mutant tumors (37 total), $37.8 \%$ were serous, $32.4 \%$ were endometrioid, $13.5 \%$ were carcinosarcoma, $8.1 \%$ were clear cell carcinoma, and $8.1 \%$ were mixed type. HNF1 $\beta$ showed similar lack of correlation with a specific histologic type with $71.9 \%$ of positive cases being endometrioid, $10.5 \%$ serous, $7.0 \%$ carcinosarcoma, $7.0 \%$ clear cell carcinoma, and $3.5 \%$ mixed type. Stated another way, $33.3 \%$ of carcinosarcomas, $66.7 \%$ of clear cell carcinomas, $13.7 \%$ of endometrioid carcinomas, $40 \%$ of mixed type tumors, and $31.6 \%$ of serous carcinomas stained positively for HNF1 $\beta$.

Mutant p53 staining had variable correlation with aberrant p16 staining, a previously reported association especially in serous cancers [23]. In our overall cohort, p53 and p16 stainings were discordant in $8.9 \%$ of cases (31/347). Of these cases, $48.3 \%$ (15/31) demonstrated mutant p53 with only patchy p16 staining.

3.4. Marker Expression Patterns. Hierarchical clustering of biomarker results from 240 cases with complete data shows 
TABLE 2: Biomarker expression by histotype.

(a) Reportable marker results in 360 tumors studied

\begin{tabular}{|c|c|c|c|c|c|c|c|c|c|c|c|c|}
\hline & \multicolumn{12}{|c|}{ Immunomarker, scored results } \\
\hline & $\begin{array}{c}\text { AMACR } \\
\text { POS }\end{array}$ & $\begin{array}{l}\text { ARID1A } \\
\text { NEG }\end{array}$ & $\begin{array}{l}\text { HNF1B } \\
\text { POS }\end{array}$ & $\begin{array}{c}\mathrm{ER} \\
>10 \%\end{array}$ & $\begin{array}{c}\text { GATA3 } \\
\text { POS }\end{array}$ & $\begin{array}{c}\text { Napsin A } \\
\text { POS }\end{array}$ & $\begin{array}{l}\text { P16 } \\
\text { POS }\end{array}$ & $\begin{array}{c}\text { P53 } \\
\text { Mutant }\end{array}$ & $\begin{array}{l}\text { PAX2 } \\
\text { NEG }\end{array}$ & $\begin{array}{c}\text { PAX8 } \\
\text { POS }\end{array}$ & $\begin{array}{c}\mathrm{PR} \\
>10 \%\end{array}$ & $\begin{array}{c}\text { PTEN } \\
\text { NEG }\end{array}$ \\
\hline Total cases stained & 360 & 360 & 360 & 360 & 360 & 360 & 360 & 360 & 360 & 360 & 360 & 360 \\
\hline Total excluded & 19 & 23 & 19 & 12 & 20 & 16 & 10 & 4 & 13 & 24 & 16 & 52 \\
\hline Total \# informative & 341 & 337 & 341 & 348 & 340 & 344 & 350 & 356 & 347 & 336 & 344 & 308 \\
\hline Total \# abnormal & 48 & 115 & 57 & 300 & 16 & 6 & 37 & 37 & 253 & 282 & 264 & 124 \\
\hline$\%$ abnormal & 14.1 & 34.1 & 16.7 & 86.2 & 4.7 & 1.7 & 10.6 & 10.4 & 72.9 & 83.9 & 76.7 & 40.3 \\
\hline
\end{tabular}

(b) Distribution of reportable marker results, by tumor histotype

\begin{tabular}{|c|c|c|c|c|c|c|c|c|c|c|c|c|}
\hline \multirow[b]{2}{*}{ Histotype } & \multicolumn{12}{|c|}{ Immunomarker scored results, informative cases \% ( $n /$ total) } \\
\hline & AMACR & ARID1A & HNF1 $\beta$ & ER & GATA3 & $\begin{array}{c}\text { Napsin } \\
\text { A }\end{array}$ & P16 & P53 & PAX2 & PAX8 & PR & PTEN \\
\hline Endometrioid & $\begin{array}{c}13.4 \% \\
(40 / 299)\end{array}$ & $\begin{array}{c}37.2 \% \\
(110 / 296)\end{array}$ & $\begin{array}{c}13.7 \% \\
(41 / 299)\end{array}$ & $\begin{array}{c}92.7 \% \\
(281 / 303)\end{array}$ & $\begin{array}{c}3.3 \% \\
(10 / 299)\end{array}$ & $\begin{array}{c}0 \% \\
(0 / 302)\end{array}$ & $\begin{array}{c}4.2 \% \\
(13 / 306)\end{array}$ & $\begin{array}{c}3.9 \% \\
(12 / 311)\end{array}$ & $\begin{array}{c}75.5 \% \\
(228 / 302)\end{array}$ & $\begin{array}{c}82.7 \% \\
(243 / 294)\end{array}$ & $\begin{array}{c}85.1 \% \\
(256 / 301)\end{array}$ & $\begin{array}{c}43.4 \% \\
(116 / 267)\end{array}$ \\
\hline Serous & $\begin{array}{c}5.3 \% \\
(1 / 19)\end{array}$ & $\begin{array}{c}5.6 \% \\
(1 / 18)\end{array}$ & $\begin{array}{l}31.6 \% \\
(6 / 19)\end{array}$ & $\begin{array}{c}55 \% \\
(11 / 20)\end{array}$ & $\begin{array}{l}15.8 \% \\
(3 / 19)\end{array}$ & $\begin{array}{c}0 \% \\
(0 / 19)\end{array}$ & $\begin{array}{c}70 \% \\
(14 / 20)\end{array}$ & $\begin{array}{c}73.7 \% \\
(14 / 19)\end{array}$ & $\begin{array}{c}55 \% \\
(11 / 20)\end{array}$ & $\begin{array}{c}95 \% \\
(19 / 20)\end{array}$ & $\begin{array}{l}26.3 \% \\
(5 / 19)\end{array}$ & $\begin{array}{l}11.8 \% \\
(2 / 17)\end{array}$ \\
\hline Carcinosarcoma & $\begin{array}{c}8.3 \% \\
(1 / 12)\end{array}$ & $\begin{array}{l}16.7 \% \\
(2 / 12)\end{array}$ & $\begin{array}{l}33.3 \% \\
(4 / 12)\end{array}$ & $\begin{array}{l}30.8 \% \\
(4 / 13)\end{array}$ & $\begin{array}{l}16.7 \% \\
(2 / 12)\end{array}$ & $\begin{array}{c}8.3 \% \\
(1 / 12)\end{array}$ & $\begin{array}{l}66.7 \% \\
(8 / 12)\end{array}$ & $\begin{array}{l}35.7 \% \\
(5 / 14)\end{array}$ & $\begin{array}{c}76.9 \% \\
(10 / 13)\end{array}$ & $\begin{array}{l}81.8 \% \\
(9 / 11)\end{array}$ & $\begin{array}{l}15.4 \% \\
(2 / 13)\end{array}$ & $\begin{array}{c}25 \% \\
(3 / 12)\end{array}$ \\
\hline $\begin{array}{l}\text { Clear cell } \\
\text { carcinoma }\end{array}$ & $\begin{array}{c}83.3 \% \\
(5 / 6)\end{array}$ & $\begin{array}{c}0 \% \\
(0 / 6)\end{array}$ & $\begin{array}{c}66.7 \% \\
(4 / 6)\end{array}$ & $\begin{array}{c}33.3 \% \\
(2 / 6)\end{array}$ & $\begin{array}{c}16.7 \% \\
(1 / 6)\end{array}$ & $\begin{array}{c}66.7 \% \\
(4 / 6)\end{array}$ & $\begin{array}{c}0 \% \\
(0 / 6)\end{array}$ & $\begin{array}{l}50 \% \\
(3 / 6)\end{array}$ & $\begin{array}{c}33.3 \% \\
(2 / 6)\end{array}$ & $\begin{array}{c}100 \% \\
(6 / 6)\end{array}$ & $\begin{array}{c}16.7 \% \\
(1 / 6)\end{array}$ & $\begin{array}{c}16.7 \% \\
(1 / 6)\end{array}$ \\
\hline Mixed type & $\begin{array}{l}20 \% \\
(1 / 5)\end{array}$ & $\begin{array}{l}40 \% \\
(2 / 5)\end{array}$ & $\begin{array}{l}40 \% \\
(2 / 5)\end{array}$ & $\begin{array}{c}33.3 \% \\
(2 / 6)\end{array}$ & $\begin{array}{c}0 \% \\
(0 / 4)\end{array}$ & $\begin{array}{l}20 \% \\
(1 / 5)\end{array}$ & $\begin{array}{c}33.3 \% \\
(2 / 6)\end{array}$ & $\begin{array}{l}50 \% \\
(3 / 6)\end{array}$ & $\begin{array}{c}33.3 \% \\
(2 / 6)\end{array}$ & $\begin{array}{l}100 \% \\
(5 / 5)\end{array}$ & $\begin{array}{c}0 \% \\
(0 / 5)\end{array}$ & $\begin{array}{c}33.3 \% \\
(2 / 6)\end{array}$ \\
\hline
\end{tabular}

* Percentages are calculated for each marker (columns) by histotype (rows). Scorable results only, noninformative cases excluded.

two main clades corresponding to PTEN and P53 mutant classes (Figure 2).

3.5. Age, Body Mass Index, and Biomarker Expression. Age at diagnosis was found to be statistically different across histologic categories $(p=0.02$, Table 3$)$, with endometrioid type tumors presenting on average at a younger age $($ mean $=68.5 y)$ than other subtypes. Body mass index did not differ significantly across diagnostic categories $(p=0.1$, Table 3).

When body mass index and age were compared with biomarker expression (Table 4), several significant associations were found. Body mass index was significantly lower in women whose tumors expressed HNF1 $\beta$ (26.9 vs. $\left.30.5 \mathrm{~kg} / \mathrm{m}^{2}, p<0.001\right)$, Napsin A $\left(25.1\right.$ vs. $30.0 \mathrm{~kg} / \mathrm{m}^{2}, p=$ $0.007)$, and mutant p53 (26.0 vs. $\left.30.3 \mathrm{~kg} / \mathrm{m}^{2}, p<0.001\right)$. Body mass index was significantly higher in women whose tumors expressed ER (30.3 vs. $\left.27.2 \mathrm{~kg} / \mathrm{m}^{2}, p=0.001\right)$ and PR (30.4 vs. $\left.28.2 \mathrm{~kg} / \mathrm{m}^{2}, p=0.033\right)$. The loss of expression of PAX2 was associated with younger age at diagnosis (68.6 vs. $70.5, p=0.035)$.

\section{Discussion}

Our study addresses two key issues. First, we performed a centralized review of the incidental endometrial cancers in the Nurses' Health Study. Secondly, through the administration of biomarkers to a large, incidental cohort, we explored biomarker expression across a spectrum of endometrial tumors with variable morphologies.

As was expected, most incidental cases of endometrial cancer in the Nurses' Health Study (87.2\% of cases) were "type 1 endometrial carcinomas" (i.e., endometrioid adenocarcinomas arising secondary to estrogen stimulation). Such "type 1" cancers account for the majority of endometrial tumors and typically arise in obese, post-menopausal women [24-26]. “Type II endometrial carcinomas" (i.e., predominantly nonendometrioid carcinomas arising in the setting of TP53 mutation or 1p deletion) [25-27], were less common in our cohort, reflecting their lower prevalence in the general population.

Our biomarker exploration of these tumors confirmed the preexisting associations between type I and type II endometrial carcinomas with hormonal receptors and p53 expression. Low-grade endometrioid carcinomas (type I) expressed high rates of ER and PR, supporting the hormonal pathway to carcinogenesis. Likewise, our type 2 tumors (nonendometrioid) demonstrated frequent p53 overexpression (mutant phenotype). Such findings are not novel, but do support the feasibility and reliability of applying biomarkers to large epidemiologic studies in a retrospective fashion.

While we confirmed preexisting biomarker expression pattern within histotypes, the advantage of our study was the ability to assess biomarkers across an incidental cohort of histomorphologic subtypes. Multiple marker-histotype correlations were found to be less specific than previously thought. Specifically, while p53 abnormalities are seen in 

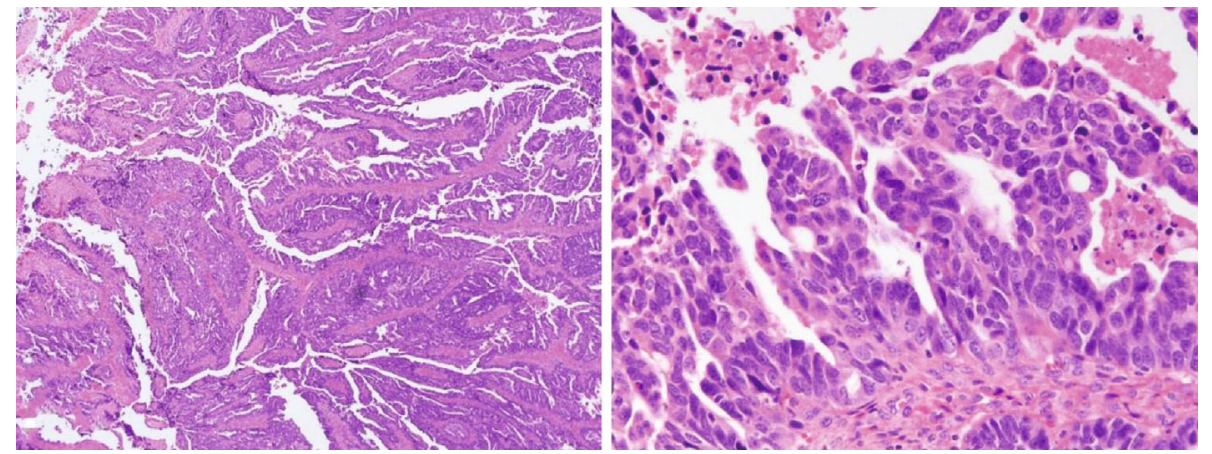

(a)
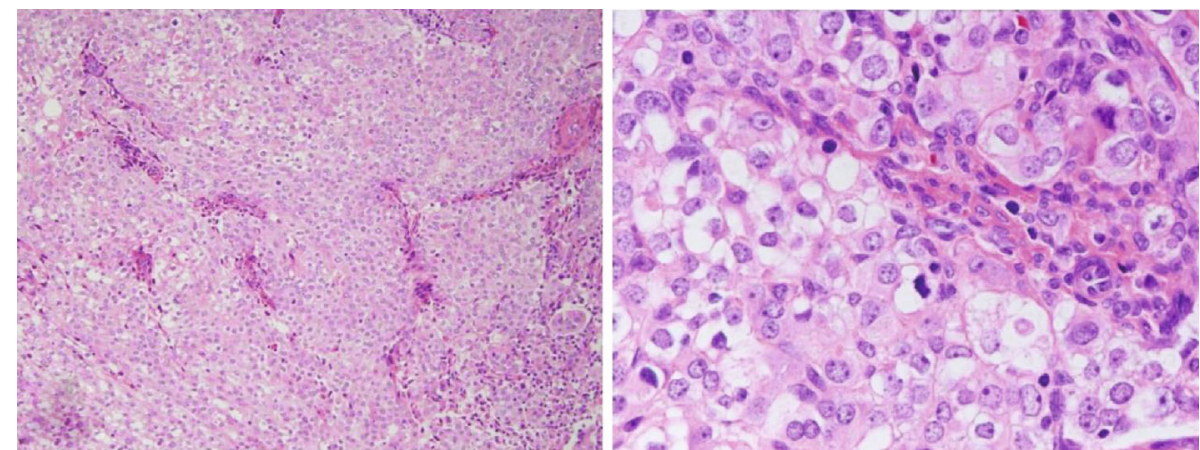

(b)
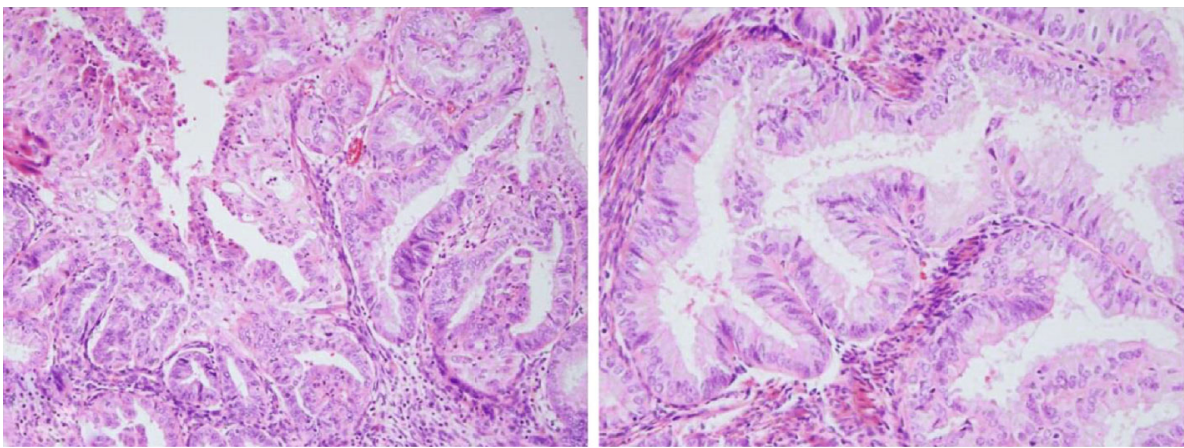

(c)

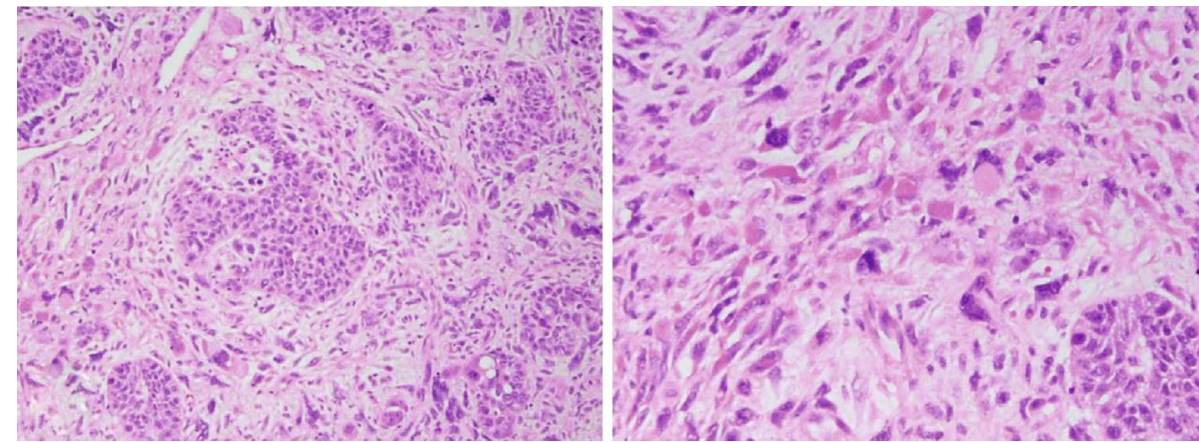

(d)

FIGURE 1: Four examples ((a-d), each a separate case) of GATA3-positive tumors with nonmesonephric histology. (a) Serous. (b) Ambiguous morphology, most consistent with grade 3 endometrioid carcinoma. (c) Conventional grade 1 endometrioid. (d) Carcinosarcoma.

$73.7 \%$ of serous cancers, $62.2 \%$ of all p53 abnormal cancers had a nonserous histology. This experience is mirrored in other purely marker-driven endometrial cancer classification systems such as the genomic-based TCGA classification schema, where molecular and histopathologic classes only partly overlap, and some unique molecular phenotypes of clinical interest (such as polymerase E mutation) cannot be reliably identified by histology alone $[8,16]$. 


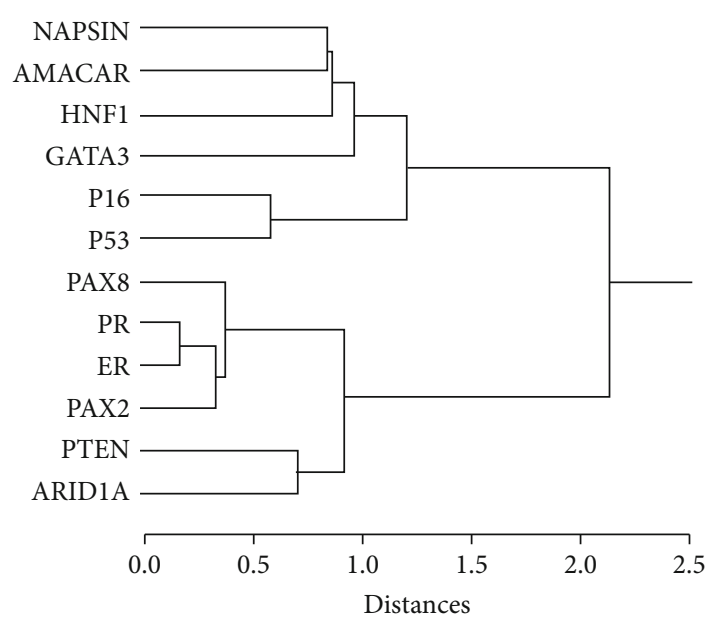

FIGURE 2: Self-organized hierarchical clustering of 12 biomarkers in 274 endometrial cancers. Dendrogram shows degree of marker association (distance measure) in 274 cases with complete data for all markers (cases with partial missing data excluded for computational reasons). Estrogen (ER) and progesterone (PR) receptor results scored dichotomously across a 10\% threshold. Two major clusters are evident, containing P53 and PTEN, respectively. Ward's linkage method and Jaccard similarity coefficient distance metric for binary data. Distal branches, such as those containing $\mathrm{ER}+\mathrm{PR}$ and $\mathrm{p} 16+\mathrm{p} 53$, indicate a level of association between members of the limb.

TABLE 3: Mean age and body mass index at presentation by tumor histotype.

\begin{tabular}{lcc}
\hline Tumor histotype & Mean age (years) & Body mass index $\left(\mathrm{kg} / \mathrm{m}^{2}\right)$ \\
\hline Endometrioid & 68.5 & 30.3 \\
Serous & 72.7 & 27.5 \\
Carcinosarcoma & 73.3 & 26.8 \\
Clear cell carcinoma & 71.4 & 25.2 \\
Mixed type & 69.8 & 28.6 \\
$p$ (Kruskal-Wallace) & 0.11 & 0.115 \\
\hline
\end{tabular}

Our study additionally contributes to the growing evidence that HNF1 $\beta$ is a nonspecific marker [15-17]. While two-thirds of our clear-cell cases stained with HNF1 $\beta$, $31.6 \%$ of serous carcinomas and $13.7 \%$ of endometrioid carcinomas expressed $\mathrm{HNF} 1 \beta$, making $\mathrm{HNF} 1 \beta$ staining an unreliable marker in the clear cell versus serous/endometrioid differential. Thus, we conclude that HNF1 $\beta$, on its own, should not be considered a specific marker for clear cell carcinoma in clinical practice.

Our findings regarding expression of GATA3 are also of interest. Prior studies demonstrate GATA3 be a highly sensitive and specific marker for mesonephric lineage in lesions of the lower female genital tract $[28,29]$. In our study, GATA3 positive staining in an unselected sample of uterine tumors did not correspond well to any single histotype. Notably, none of the cases, even those of low-grade cytology, displayed the classic morphologic features of mesonephric carcinomas (e.g., small round tubules, cuboidal or flatted epithelium, and angulated vesicular nuclei). Furthermore, 12 of 16 positive cases displayed high-grade or ambiguous cytologic features, raising the possibility that poorly differentiated endometrial tumors may express GATA3 not due to lineage but rather due to gain of function mutations. This is further demonstrated by our finding that $4.6 \%$ of cases demonstrated positivity for both PAX8 and GATA3, two stains that should be mutually exclusive of each other.

An unsupervised self-organizing dendrogram of marker results in our tumors gives an indication of how combinatorial marker trends can define tumor subgroups (Figure 2). Two major clusters are evident: a p53 mutant arm and a PTEN/PAX2 arm, corresponding to "type II" and "type I" endometrial cancers, respectively. Further, there is a p16/p53 subgroup distinct from a Napsin A/AMACR/ HNF1 $\beta$ class. Of note, GATA3 expression clustered with both clear cell markers and/or p53/p16 expression. This clustering provides additional evidence that GATA3 expression likely denotes aberrant expression/gain of function mutations in high-grade malignancies arising from mutations in TP53 rather than mesonephric lineage.

Given our unique dataset, we were able to compare biomarker expression across participants' ages and body mass index. As expected, p53 expression was associated with significantly lower body mass index, and ER/PR expression was associated with significantly higher body mass index (additional ER/PR findings in this cohort are reported elsewhere [20]). Additionally, endometrioid-type tumors tended to present at earlier ages than other subtypes. These findings are consistent with common mechanisms of endometrial carcinogenesis in which either hormonal stimulation (typically in the setting of obesity) [30] or p53 mutation leads to neoplastic growth $[25,26,31,32]$.

Our study did highlight some of the necessities and limitations of working with tissue microarrays. Of note, when performing a centralized pathology review, we found it essential to render a morphologic diagnosis on whole $\mathrm{H} \& \mathrm{E}$ slides only, not on the tissue microarray preparations. This is due to the limited amount of tumor present on the tissue microarray. Secondly, due to the occasional absence of internal controls noted in the tissue microarray tissues, we recommend running separate positive controls on each tissue microarray assay. We also recommend replicate stain reads to control for idiosyncratic runs, ideally with the replicates reviewed independently by mutually blinded pathologists. The main limitation of using tissue microarrays in our study was the inability to reliably interpret $\mathrm{p} 53$ protein null phenotypes caused by rare nonsense mutations [33]. Thus, more of our cases likely had aberrant p53 staining than we could reliably conclude from our tissue microarray preparations.

In this study, we provide a centralized review of the incidental endometrial cancers in the Nurses' Health Study as well as the biomarker expression results across this cohort. This data is largely applicable to future clinical/epidemiologic studies that derive data on endometrial cancers form the Nurses' Health Study. However, we were also able to test the hypothesis that biomarker studies in which cases are preselected by pathologists likely lead to overestimated sensitivities and specificities. In the case of GATA3, p53, and 
Table 4: Mean Age and Body Mass Index at Presentation by Marker Status (p, t-test separate variance).

\begin{tabular}{lccccccc}
\hline & Age & Age & Age & Body mass index & Body mass index & Body mass index & Comment \\
\hline Marker & Wild type & Mutant & $p$ & Wild type & Mutant & $p$ & 0.923 \\
AMACR & 69.0 & 68.7 & 0.775 & 29.8 & 30.0 & 29.2 & 0.160 \\
ARID1A & 69.2 & 68.6 & 0.539 & 30.3 & 26.9 & 25.1 & 0.007 \\
HNF1 $\beta$ & 68.8 & 69.9 & 0.354 & 30.5 & 29.5 & 0.771 & 0.001 \\
Napsin A & 68.9 & 73.0 & 0.357 & 29.9 & 30.3 & 0.033 \\
p16 & 68.9 & 70.5 & 0.178 & 30.0 & 30.4 & 0.067 \\
ER & 70.7 & 68.8 & 0.111 & 27.2 & 29.5 & 0.252 \\
PR & 69.6 & 68.8 & 0.402 & 28.3 & 30.1 & $<0.001$ \\
PAX8 & 69.0 & 69.0 & 1.000 & 31.6 & 26.0 & 0.979 \\
PAX2 & 70.5 & 68.6 & 0.035 & 29.1 & 29.9 & 0.108 \\
p53 & 68.9 & 70.3 & 0.336 & 30.3 & 26.9 & is wild type \\
PTEN & 69.9 & 68.4 & 0.077 & 29.8 & 30.0 & & \\
GATA3 & 68.9 & 71.1 & 0.290 & & & \\
\hline
\end{tabular}

HNF1 $\beta$, we found this hypothesis to be true. We believe our approach of testing biomarkers across an incidental population of cancers gives a more realistic sense of the utility of such markers when applied to incidental disease. Further, our findings mimic those of The Cancer Genome Atlas and other data collected from next-generation sequencing in which there is much overlap in the mutational profiles of tumor categories as defined by histomorphology. We conclude that expression of a single biomarker (e.g., HNF1 $\beta$ and GATA3) should not be interpreted as diagnostic of a particular tumor type without taking into consideration the histology. We would recommend that, when in doubt of the histology, a panel of immunostains, including stains redundant for a diagnosis (e.g., PTEN, ARID1a, and ER for endometrioid adenocarcinoma; HNF1 $\beta$, Napsin A, and AMACR for clear cell carcinoma; and p16 and p53 for serous carcinoma), be performed rather than relying on a single immunostain. Lastly, we show that tissue microarrays have some limitations; however, the results of biomarker studies in this setting are largely reliable and reproducible.

\section{Data Availability}

The Nurses' Health Study data are housed within the Harvard School of Public Health and are not publicly available without an IRB and application for access.

\section{Additional Points}

Highlights. (1) This study characterizes all incidental endometrial cancers in the Nurses' Health Study. (2) Our findings call into question specificity of several commonly used biomarkers. (3) We demonstrate that tissue microarrays are reliable and reproducible in retrospective studies.

\section{Disclosure}

The funders had no role in study design, data collection and analysis, decision to publish, or preparation of the manuscript. The corresponding author had full access to all the data in the study and had final responsibility for the decision to submit for publication. Jaclyn C. Watkins present address is Department of Pathology, Massachusetts General Hospital, 55 Fruit St., Boston, MA 02114, USA.

\section{Conflicts of Interest}

The authors have no conflicts of interest to report.

\section{Authors' Contributions}

Jaclyn C. Watkins is the primary author and involved in immunohistochemical and statistical data collection, analyses, and interpretation. Michael J. Downing is involved technically in tissue microarray construction and immunohistochemical staining and provided edits to the final manuscript. Marta Crous-Bou is involved in epidemiologic portion of data collection and provided edits to final manuscript. Evan L. Busch is involved in epidemiologic portion of data collection and provided edits to the final manuscript. Maxine Chen is involved in epidemiologic portion of data collection and provided edits to the final manuscript. Immaculata De Vivo is the principal collaborator from the Nurses' Health Study and assisted in the study design and edits to the final manuscript. George L. Mutter is the principal investigator; designed study, collected data, and performed statistical analyses; and provided final edits to the manuscript. All authors have approved the final article.

\section{Acknowledgments}

The authors would like to thank the participants and staff of the Nurses' Health Study for their valuable contributions as well as the following state cancer registries for their help: $\mathrm{AL}, \mathrm{AZ}, \mathrm{AR}, \mathrm{CA}, \mathrm{CO}, \mathrm{CT}, \mathrm{DE}, \mathrm{FL}, \mathrm{GA}, \mathrm{ID}, \mathrm{IL}, \mathrm{IN}, \mathrm{IA}, \mathrm{KY}$, LA, ME, MD, MA, MI, NE, NH, NJ, NY, NC, ND, OH, OK, OR, PA, RI, SC, TN, TX, VA, WA, and WY. We also thank the Channing Division of Network Medicine, Department of Medicine, Brigham and Women's Hospital, and Harvard Medical School. Finally, we would like to 
acknowledge the members of the De Vivo and Mutter laboratories for their assistance. The Nurses' Health Study is supported by the National Cancer Institute-National Institutes of Health (UM1 CA186107 and P01 CA87969). This study was supported by a grant from the National Cancer Institute (2 R01 CA082838-10). ELB and MMC were supported in part by a grant from the National Cancer Institute (5T32CA009001). Additional project support was provided by Departmental (Pathology, BWH) Research Funds (GLM).

\section{References}

[1] G. L. Mutter and J. Prat, "Endometrial Adenocarcinoma," in Pathology of the Female Reproductive Tract, pp. 370-401, Elsevier, 2014.

[2] R. Zaino, S. Carinelli, and L. Ellenson, "No Title," WHO Classification of Tumours of the Female Reproductive Organs, R. J. Kurman, M. Cacangiu, S. Herrington, and R. Young, Eds., 125-134, 2014.

[3] L. N. Hoang, M. A. Kinloch, J. M. Leo et al., "Interobserver agreement in endometrial carcinoma histotype diagnosis varies depending on The Cancer Genome Atlas (TCGA)-based molecular subgroup," The American Journal of Surgical Pathology, vol. 41, no. 2, pp. 245-252, 2017.

[4] L. Lomo, M. R. Nucci, K. R. Lee et al., "Histologic and immunohistochemical decision-making in endometrial adenocarcinoma," Modern Pathology, vol. 21, no. 8, pp. 937-942, 2008.

[5] C. B. Gilks, E. Oliva, and R. A. Soslow, "Poor interobserver reproducibility in the diagnosis of high-grade endometrial carcinoma," The American Journal of Surgical Pathology, vol. 37, no. 6, pp. 874-881, 2013.

[6] Y. R. Hussein, R. Broaddus, B. Weigelt, D. A. Levine, and R. A. Soslow, "The genomic heterogeneity of FIGO grade 3 endometrioid carcinoma impacts diagnostic accuracy and reproducibility," International journal of gynecological pathology, vol. 35, no. 1, pp. 16-24, 2016.

[7] G. Han, D. Sidhu, M. A. Duggan et al., "Reproducibility of histological cell type in high-grade endometrial carcinoma," Modern pathology, vol. 26, no. 12, pp. 1594-1604, 2013.

[8] Cancer Genome Atlas Research Network, C. Kandoth, N. Schultz et al., "Integrated genomic characterization of endometrial carcinoma," Nature, vol. 497, no. 7447, pp. 6773, 2013.

[9] M. E. Barnard, A. Pyden, M. S. Rice et al., "Inter-pathologist and pathology report agreement for ovarian tumor characteristics in the Nurses' Health Studies," Gynecologic oncology, vol. 150, no. 3, pp. 521-526, 2018.

[10] S. Hu, J. L. Hinson, R. Matnani, M. L. Cibull, and R. G. Karabakhtsian, "Are the uterine serous carcinomas underdiagnosed? Histomorphologic and immunohistochemical correlates and clinical follow up in high-grade endometrial carcinomas initially diagnosed as high-grade endometrioid carcinoma," Modern Pathology, vol. 31, no. 2, pp. 358-364, 2018.

[11] N. Kato, S. Sasou, and T. Motoyama, "Expression of hepatocyte nuclear factor-1beta (HNF-1beta) in clear cell tumors and endometriosis of the ovary," Modern Pathology, vol. 19, no. 1, pp. 83-89, 2006.

[12] S. Yamamoto, H. Tsuda, S. Aida, H. Shimazaki, S. Tamai, and O. Matsubara, "Immunohistochemical detection of hepatocyte nuclear factor 1beta in ovarian and endometrial clear-cell ade- nocarcinomas and nonneoplastic endometrium," Human Pathology, vol. 38, no. 7, pp. 1074-1080, 2007.

[13] D. DeLair, E. Oliva, M. Köbel, A. Macias, C. B. Gilks, and R. A. Soslow, "Morphologic spectrum of immunohistochemically characterized clear cell carcinoma of the ovary," The American Journal of Surgical Pathology, vol. 35, no. 1, pp. 36-44, 2011.

[14] D. DeLair, G. Han, J. Irving et al., "HNF-1 $\beta$ in ovarian carcinomas with serous and clear cell change," International Journal of Gynecological Pathology, vol. 32, no. 6, pp. 541-546, 2013.

[15] Y.-C. Kao, M.-C. Lin, W.-C. Lin, Y.-M. Jeng, and T.-L. Mao, "Utility of hepatocyte nuclear factor- $1 \beta$ as a diagnostic marker in ovarian carcinomas with clear cells," Histopathology, vol. 61, no. 5, pp. 760-768, 2012.

[16] L. N. Hoang, G. Han, M. McConechy et al., "Immunohistochemical characterization of prototypical endometrial clear cell carcinoma-diagnostic utility of HNF-1 $\beta$ and oestrogen receptor," Histopathology, vol. 64, no. 4, pp. 585-596, 2014.

[17] K. Němejcová, I. Tichá, P. Kleiblová et al., "Expression, epigenetic and genetic changes of HNF1B in endometrial lesions," Pathology Oncology Research, vol. 22, no. 3, pp. 523-530, 2016.

[18] G. Colditz and S. E. Hankinson, "The Nurses' Health Study: lifestyle and health among women," Nature Reviews. Cancer, vol. 5, no. 5, pp. 388-396, 2005.

[19] C. F. Belanger, C. H. Hennekens, B. Rosner, and F. E. Speizer, "The nurses' health study," The American Journal of Nursing, vol. 78, no. 6, pp. 1039-1040, 1978.

[20] E. L. Busch, M. Crous-Bou, J. Prescott et al., "Endometrial cancer risk factors, hormone receptors, and mortality prediction," Cancer Epidemiology and Prevention Biomarkers, vol. 26, no. 5, pp. 727-735, 2017.

[21] T. A. Longacre, R. Broaddus, L. T. Chuang et al., "Template for reporting results of biomarker testing of specimens from patients with carcinoma of the endometrium," Archives of Pathology \& Laboratory Medicine, vol. 141, no. 11, pp. 1508$1512,2017$.

[22] E. B. Rimm, M. J. Stampfer, G. A. Colditz, C. G. Chute, L. B. Litin, and W. C. Willett, "Validity of self-reported waist and hip circumferences in men and women," Epidemiology, vol. 1, no. 6, pp. 466-473, 1990.

[23] K. Z. Hanley, G. G. Birdsong, and M. B. Mosunjac, "Recent developments in surgical pathology of the uterine corpus," Archives of Pathology \& Laboratory Medicine, vol. 141, no. 4, pp. 528-541, 2017.

[24] R. W. O’Rourke, "Endometrial hyperplasia, endometrial cancer, and obesity: convergent mechanisms regulating energy homeostasis and cellular proliferation," Surgery for Obesity and Related Diseases, vol. 10, no. 5, pp. 926-928, 2014.

[25] P. B. Clement and R. H. Young, "Endometrioid carcinoma of the uterine corpus: a review of its pathology with emphasis on recent advances and problematic aspects," Advances in Anatomic Pathology, vol. 9, no. 3, pp. 145-184, 2002.

[26] J. V. Bokhman, "Two pathogenetic types of endometrial carcinoma," Gynecologic Oncology, vol. 15, no. 1, pp. 10-17, 1983.

[27] M. Hendrickson, J. Ross, P. Eifel, A. Martinez, and R. Kempson, "Uterine papillary serous carcinoma: a highly malignant form of endometrial adenocarcinoma," The American Journal of Surgical Pathology, vol. 6, no. 2, pp. 93-108, 1982.

[28] B. E. Howitt, M. M. Emori, R. Drapkin et al., "GATA3 is a sensitive and specific marker of benign and malignant mesonephric lesions in the lower female genital tract," The American 
Journal of Surgical Pathology, vol. 39, no. 10, pp. 1411-1419, 2015.

[29] A. A. Roma, A. Goyal, and B. Yang, "Differential expression patterns of GATA3 in uterine mesonephric and nonmesonephric lesions," International Journal of Gynecological Pathology, vol. 34, no. 5, pp. 480-486, 2015.

[30] S. Mahabir, D. J. Baer, L. L. Johnson et al., "Usefulness of body mass index as a sufficient adiposity measurement for sex hormone concentration associations in postmenopausal women," Cancer Epidemiology, Biomarkers \& Prevention, vol. 15, no. 12, pp. 2502-2507, 2006.

[31] L. Deligdisch and C. F. Holinka, "Endometrial carcinoma: two diseases?," Cancer Detection and Prevention, vol. 10, no. 3-4, pp. 237-246, 1987.

[32] M. E. Sherman, "Theories of endometrial carcinogenesis: a multidisciplinary approach," Modern Pathology, vol. 13, no. 3, pp. 295-308, 2000.

[33] B. Murnyák and T. Hortobágyi, "Immunohistochemical correlates of TP53 somatic mutations in cancer," Oncotarget, vol. 7, no. 40, pp. 64910-64920, 2016. 